\title{
Chromosomal locations of twelve isozyme loci in Pisum sativum
}

N. F. Weeden
G. A. Marx

The authors are affiliated with the Department of Horticultural Sciences, New York Agricultural Experiment Station, Cornell University, Geneva, NY 14456. Invaluable technical assistance was provided in these studies by A. C. Emmo. The authors also thank D. W. Barton and R. W. Robinson for their helpful comments and reviews.

1984, American Genetic Association.

ABSTRACT: Approximate chromosomal locations of 12 loci specifying electrophoretic enzyme variants are described in the garden pea (Pisum sativum L.). The enzyme loci are distributed on five of the seven chromosomes. The position of the loci on chromosomes 2 and 3 are such that most of the known markers on these chromosomes will exhibit linkage with at least one of the isozyme loci. Several of the loci studied code for enzymes that have isozymic counterparts in other compartments of the cell. In order to distinguish among the genes coding these isozymes we have added a suffix to the locus designation corresponding to the intracellular location of its product.

ISOZYMES are distinguishable forms of an enzyme often found within the same cell. In many cases the variability can be attributed to different alleles at a single locus, identifying the variants as a specific subclass of isozymes called allozymes. The simple genetic basis of allozymic forms permits their use as genetic markers for a number of applications ${ }^{24}$. Mapping of these "isozyme loci" in maize", tomatoes ${ }^{18}$, barley 6 , wheat and related species ${ }^{13,14}$ has resulted in the formation of partial linkage maps, extremely useful tools for the breeder and plant geneticist. Conspicuously missing from the list of plants investigated are representatives from the Leguminosae, a major family of flowering plants both in terms of number of taxa and importance to agriculture. Initial genetic studies of the isozymes in soybeans ${ }^{10.11}$, alfalfa ${ }^{17}$, and beans ${ }^{3.26}$ have been published, but the loci have not been mapped.

For several reasons the garden pea is highly suited for the development of an enzyme linkage map. It already possesses many chromosomal markers that facilitate mapping of isozyme loci; it is a convenient plant for genetic studies (short generation time, naturally self-pollinated and easily cross pollinated, diverse germplasm collections available); and it may possibly serve as a model system for other commercially important legumes. At present, only one isozyme locus has been mapped on the pea genome, Lap-I, which codes for the more anodal leucine aminopeptidase isozyme observed after starch gel electrophoresis of raw extracts ${ }^{2}$. However, a large number of polymorphic isozyme loci have been identified ${ }^{27}$, potentially enough to form an enzyme linkage map covering the entire genome should the loci be distributed randomly. We have therefore initiated a program to systematically map all known polymorphic enzyme loci in the pea. We report here approximate map positions of 12 isozyme loci located on five different chromosomes.

\section{Materials and Methods}

Inbred lines of Pisum sativum with known morphological markers were selected from the pea collection available at the New York State Agricultural Experiment Station, Geneva, NY. Lines were screened for isozyme phenotype, and appropriate crosses were made with suitable marker lines. Three crosses were selected for further screening of $F_{2}$ populations, the three families being designated $A, E$, and $G$. The A family consisted of seed from six $F_{1}$ plants produced in a cross between lines B77-254 and A78-237. Three of the $F_{1}$ individuals were generated using $B 77-254$ as the female parent; the other three were from a reciprocal cross. The $F_{2}$ population, E, was derived from the cross A 1078-234 $\times$ B 77-257 while $G$ was derived from the cross B78-288 $\times$ A 1078-236. The relevant morphological markers possessed by each line are given in Table I.

Linkage between loci was calculated using the tables published by Allard' and computer 
programs "F2" and "Progeny" developed at the New York State Agricultural Experiment Station.

Samples were prepared for electrophoresis using two extraction buffers as described previously ${ }^{30}$. Samples extracted in the tris$\mathrm{HCl}$ buffer were placed on a $\mathrm{pH} 8.1$ tris-citrate/lithium borate system ${ }^{21}$. The phosphate extraction buffer was used for samples subjected to electrophoresis on either a $\mathrm{pH} 6.5$ histidine ${ }^{7}$ or a $\mathrm{pH} 6.1$ citrate/N-(3-aminopropyl)-morpholine ${ }^{8}$ gel system.

The assays for NADP-specific isocitrate dehydrogenase (IDH), acid phosphatase (AcP), 6-phosphogluconate dehydrogenase (6PGD), and leucine aminopeptidase (LAP) were identical to or slight modifications of those described by Shaw and Prasad ${ }^{22}$. The assays for aspartate aminotransferase (AAT), shikimate dehydrogenase (SKDH), aldolase (Aldo), and phosphoglucomutase (PGM) have been described previously ${ }^{3 !}$. Methylumbelliferyl esterase (Est) was assayed as described by Bender et al. ${ }^{4}$. The enzymes AAT, PGM, LAP, and SKDH were assayed on anodal slices cut from gels run using the tris-borate buffer system. The histidine buffer system was used for 6PGD, and the citrate/ $\mathrm{N}$-(3-aminopropyl)-morpholine buffer system was used to resolve the IDH, AcP, and Est isozymes.

Reagents were obtained from Sigma Chemical Company, St. Louis, Missouri, except for the $\mathrm{N}$-(3-aminopropyl) morpholine, which was purchased from Aldrich Chemical Company, Milwaukee, Wisconsin.

\section{Results}

All plants remained healthy throughout the experiment and seed set was excellent for families A and E. Some sterility was encountered among plants in the $G$ population. If this sterility was caused by differences in parental karyotype such differences did not appear to interfere with the mapping of isozyme loci, for the chromosomal markers and isozyme phe-

Table I. Relevant morphological marker genes in parental lines

\begin{tabular}{ll}
\hline \multicolumn{1}{c}{ Line } & \multicolumn{1}{c}{ Marker genes* } \\
\hline A 1078-234 & $i, s, w b, k, s t, b, n, f a$, \\
& $l e, c p, g p, w l o, t l, r$ \\
A 1078-236 & $k, M, s t, b, l e, w l o$ \\
A78-237 & $s, w b, k, s t, b, t l, r$ \\
B77-254 & $P u r, o h, P u, g p$ \\
B77-257 & $N p, B t$ \\
B78-288 & $d, a r, U, P l$ \\
\hline
\end{tabular}

* For description of characters see Blixt ${ }^{5}$

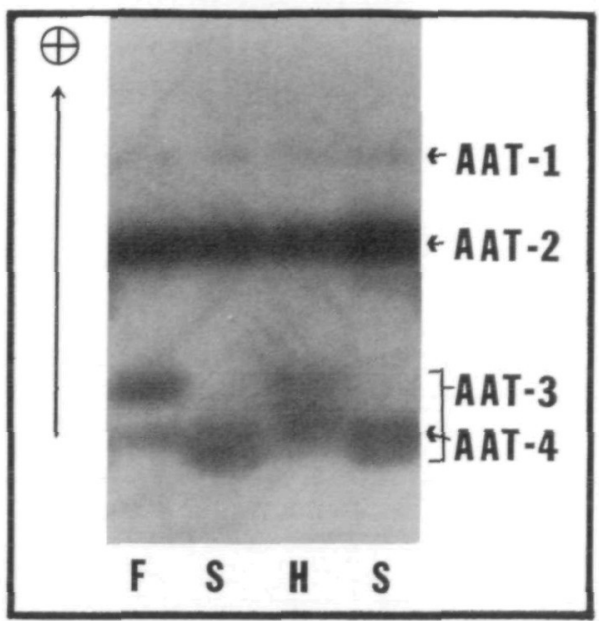

FIGURE 1 Mitochondrial aspartate aminotransferase (AAT-3) phenotypes after electrophoresis of leaf extracts. The fast alloxyme (F) has mobility approrimately intermediate between the plastid isozyme (AAT-2) and the cytosolic form (AAT-4). The slow alloxyme (S) is slower than but partially overlaps AAT-4. The heterozygous pattern is designated H. Arrow indicates direction of migration.

notypes gave normal segregation ratios, and unexpected linkages between morphological markers were not observed. Segregation ratios for the reciprocal crosses in family $A$ gave similar ratios (data not shown); therefore, the two data sets were combined for linkage analysis.

\section{Phenotypes observed after electrophoresis}

Isocitrate dehydrogenase: A single band of IDH activity was observed in extracts from inbred lines. Crosses made between lines exhibiting forms with different electrophoretic

\section{$\odot$}

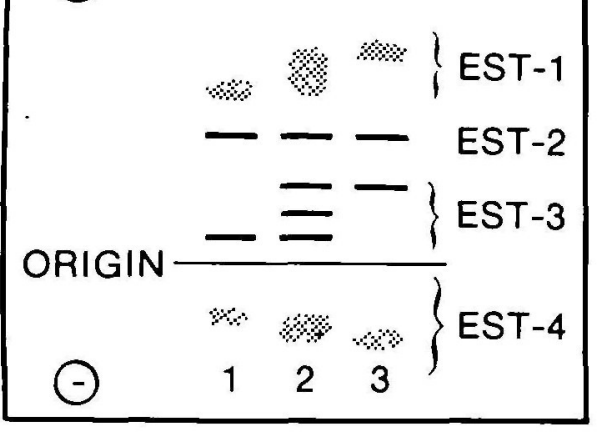

FIGURE 2 Pea leaf esterase yymograms as visualized using the florogenic substrate methylumbelliferyl acetate. Column 1 represents an extract containing the slow alloryme of each esterase. Column 2 depicts the pattern observed in a plant heteroxygous at Est-1, Est-3, and Est -4 (Est-2 is homoxygous for the slow allele). The third track shows the fast allozyme for all esterases except for Est-2, which is slow.

mobilities gave progeny with a three-banded phenotype, reflecting the dimeric structure of this enzyme. In the crosses reported here the mobilities of the two homodimeric forms did not differ enough to permit the resolution of the intermediate heterodimer.Thus, heterozygous plants displayed a single, wide band after electrophoresis.

Aspartate aminotransferase: Cell fractionation studies of the AAT isozymes in the pea indicated that the third most anodal set of allozymes, AAT-3 (Figure 1), represented mitochondrial forms. In a previous paper ${ }^{31}$ the mitochondrial specific forms were labeled 'AAT-2' because the most anodal set of AAT isozymes (AAT-1 in Figure 1) could not be seen. The fast variant possessed a mobility

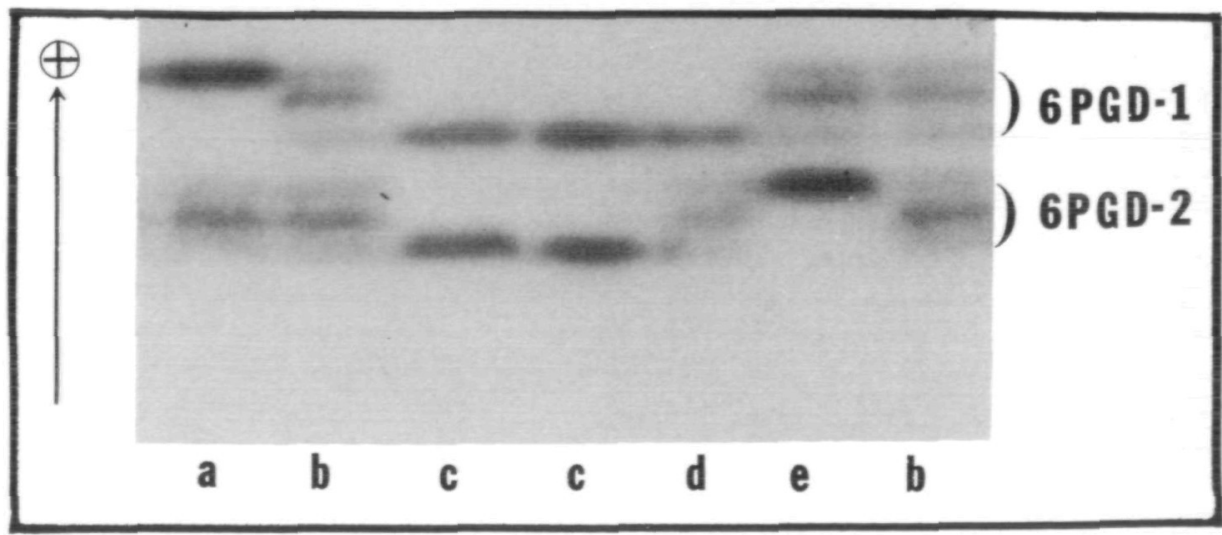

FIGURE 3 Variation observed in both isozymes of 6PGD. The phenotypes (6PGD-1/6PGD-2) corresponding to the labeled tracks are: (a) $F / H,(b) H / H$, (c) $S / S$, (d) $S / H$, and (e) $H / F$, where $F=$ fast atlozyme, $\mathbf{S}$ a slow allozyme, $\mathbf{H}=$ heterozygous phenotype. Doubly heterozygous individuals express sir bands: the four homodimeric forms and two intragenic heterodimers. 
relative to the borate front $\left(R_{f}\right)$ of 0.32 .The slow form, partially obscured by cytosolic AAT isozyme, had an $R_{f}$ of 0.25 . Heterozygous individuals exhibited a wide blur with fainter staining of the homodimeric forms.

Leucine aminopeptidase: Several investigators $^{16,20}$ have described polymorphism at two leucine aminopeptidase loci, Lap-I and Lap-2, the loci being numbered in order of decreasing migration rate of the respective isozymes. Scandalios and Espiritu ${ }^{20}$ observed that the two aminopeptidases they isolated from pea extracts could be distinguished on the basis of substrate specificity. Although both isozymes could cleave either leucyl or alanyl $\alpha$-naphthylamide, one was more active with the former substrate while the other exhibited the opposite preference. When we assayed duplicate slices from a $\mathrm{pH} 8.1$ gel for leucine aminopeptidase and alanyl aminopeptidase activity we found that both LAP-1 and LAP-2 isozymes were more active with the leucyl $\alpha$-naphthylamide substrate. However, a third set of bands, partially overlapping the LAP-2 allozymes, were visualized when alanyl $\alpha$-naphthylamide was used as the substrate. These alanyl aminopeptidase bands are products of a locus distinct from Lap-I and Lap-2 although the chromosomal location of this locus has not been determined (Weeden, unpub.). All three aminopeptidases can be clearly separated using the histidine gel system; however, the resolution of the LAP-1 allozymes is poorer on this gel.

Esterases: We found that the esterases in young leaf tissue were best observed using the florogenic substrate 4-methylumbelliferyl acetate. The citrate/3 amino-morpholine system resolved four areas of esterase activity, one cathodal and three anodal (Figure 2). The cathodal esterase, Est-4, gave relatively blurry bands, and the heterozygous pattern could only be interpreted as a broad smear.

6-Phosphogluconate dehydrogenase: Two loci are responsible for the 6PGD activity observable in the pea leaf extracts ${ }^{28}$. The more anodal isozyme (6PGD-1) is localized in the plastid compartment while 6PGD-2 is cytosolic. Plants heterozygous at either locus exhibit intragenic heterodimers (Figure 3) concordant with the dimeric structure of this enzyme ${ }^{23}$. Hybrid enzymes containing one 6PGD-1 subunit and one 6PGD-2 subunit were not observed.

Phenotypes for shikimate dehydrogenase, phosphoglucomutase, aldolase, and acid phosphatase were described previously ${ }^{31,32}$.

\section{Linkages observed}

Tables II and III present the segregation data for individual loci and joint segregation
Table II. Segregation of alleles at loci involved in mapping experiments

\begin{tabular}{|c|c|c|c|c|c|}
\hline \multirow[b]{2}{*}{ Family } & \multirow[b]{2}{*}{ Locus } & \multicolumn{3}{|c|}{ No. observed with designated phenotype } & \multirow[b]{2}{*}{$x^{2}$} \\
\hline & & $\begin{array}{l}\text { recessive } \\
\text { or slow }\end{array}$ & heterozygous & $\begin{array}{c}\text { dominant } \\
\text { or fast }\end{array}$ & \\
\hline \multirow[t]{20}{*}{ A } & Pur & 19 & & 44 & 0.89 \\
\hline & $O h$ & 23 & & 61 & 0.25 \\
\hline & $S$ & 15 & & 69 & 2.28 \\
\hline & $\boldsymbol{W b}$ & 22 & & 63 & 0.04 \\
\hline & $\boldsymbol{K}$ & 25 & & 59 & 1.02 \\
\hline & $S t$ & 22 & & 62 & 0.06 \\
\hline & $B$ & 21 & & 63 & 0.0 \\
\hline & $G p$ & 20 & & 64 & 0.06 \\
\hline & $R$ & 19 & & 65 & 0.25 \\
\hline & $T l$ & 24 & 37 & 24 & 1.42 \\
\hline & Idh & 20 & 45 & 19 & 0.45 \\
\hline & $S k d h$ & 23 & 42 & 19 & 0.38 \\
\hline & Aldo-p & 22 & 35 & 13 & 2.31 \\
\hline & $A a t-m$ & 28 & 40 & 17 & 3.14 \\
\hline & $P g m-p$ & 23 & 45 & 16 & 1.60 \\
\hline & Lap-1 & 24 & 37 & 23 & 1.21 \\
\hline & $A c p-1$ & 21 & 37 & 26 & 1.78 \\
\hline & $A c p-2$ & 21 & 33 & 21 & 1.08 \\
\hline & $A c p-3$ & 17 & 31 & 19 & 0.49 \\
\hline & $6 p g d-p$ & 23 & 39 & 23 & 0.29 \\
\hline \multirow[t]{14}{*}{$\mathbf{E}$} & $W b$ & 31 & & 85 & 0.18 \\
\hline & $K$ & 33 & & 82 & 0.84 \\
\hline & $S t$ & 36 & & 81 & 2.08 \\
\hline & $B$ & 31 & & 85 & 0.18 \\
\hline & $G p$ & 32 & & 84 & 0.40 \\
\hline & $R$ & 26 & & 84 & 0.11 \\
\hline & $T l$ & 28 & 55 & 34 & 0.77 \\
\hline & Bt & 37 & & 77 & 3.38 \\
\hline & $P g m-p$ & 25 & 52 & 32 & 1.11 \\
\hline & Lap.I & 32 & 56 & 26 & 0.67 \\
\hline & $A C p-1$ & 27 & 58 & 31 & 0.28 \\
\hline & $A C p-2$ & 29 & 49 & 29 & 0.76 \\
\hline & $6 p g d-c$ & 29 & 57 & 31 & 0.15 \\
\hline & Est -4 & 28 & 52 & 28 & 0.22 \\
\hline \multirow[t]{16}{*}{ G } & $D$ & 11 & & 29 & 0.13 \\
\hline & $A r$ & 8 & & 26 & 0.04 \\
\hline & $\kappa$ & 9 & & 30 & 0.08 \\
\hline & $M$ & 6 & & 32 & 1.80 \\
\hline & $S t$ & 12 & & 29 & 0.40 \\
\hline & $B$ & 13 & & 26 & 1.44 \\
\hline & $I d h$ & 9 & 20 & 11 & 0.20 \\
\hline & Aat-m & 10 & 23 & 8 & 0.80 \\
\hline & Skdh & 8 & 20 & 12 & 0.80 \\
\hline & Pgm-p & 8 & 17 & 16 & 4.32 \\
\hline & Lap-I & 9 & 18 & 13 & 1.20 \\
\hline & Lap-2 & 8 & 21 & 10 & 0.44 \\
\hline & $A c p-1$ & 8 & 25 & 7 & 2.55 \\
\hline & Acp-2 & 8 & 13 & 10 & 1.06 \\
\hline & Acp-3 & 7 & 20 & 9 & 0.67 \\
\hline & Est-4 & 6 & 20 & 13 & 2.54 \\
\hline
\end{tabular}


data between pairs of loci that gave significant deviations from random assortment. The morphological characters used for mapping the isozyme loci have well-characterized phenotypes and are specified by single loci (Table I) that have been located on the chromosomal map of the peas. The enzyme loci mentioned in the tables can be placed in five linkage groups, corresponding to regions of chromosomes 1, 2, 3, 5, and 7 (Figure 4).

\section{Chromosome 1}

Isocitrate dehydrogenase: The locus coding subunits of this enzyme, $I d h$, showed close linkage with two marker loci on chromosome 1: Pur and $D$. Each marker locus exhibited about 5 percent recombination with $I d h$. An 8 percent recombination frequency has been reported between $P u r$ and $D$, suggesting that Idh may be located between these two; however, a three-point test involving the loci was not performed in our experiments.

\section{Chromosome 2}

Shikimate dehydrogenase: In the A population (84 individuals) no recombinants were found between $S k d h$ and $O h$, the locus controlling a reddish-brown pigment in the testa. The positioning of $S k d h$ on chromosome 2 was further substantiated in population $\mathrm{G}$ indicating a linkage between this locus and $A r$, also located on that chromosome.

Aspartate aminotransferase: The locus specifying mitochondrial AAT, $A a t-m$, also was assigned to chromosome 2 by virtue of its linkage with $S k d h$ in both populations $A$ and G, with $O h$ in A and with $A r$ in G. The two independent determinations of linkage between $S k d h$ and $A a t-m$ gave recombination frequencies of 24 percent and 18 percent (Table III). In G a three-point test suggested that the sequence $A a t-m-S k d h-A r$ is the correct order of the genes, with Aat-m positioned toward the end of the chromosome.

Aldolase: Variation in the plastid specific aldolase was produced by alleles at locus Aldo-p, near the end of the known linkage map. In population A, Aldo-p showed linkage with both $S k d h$ and $A a t-m$, with the relative recombination frequencies indicating a gene order of $S k d h-A a t-m-A l d o-p$. These results confirm an earlier report of possible linkage between $S k d h$ and $A l d o-p^{31}$.

Phosphoglucomutase: The gene coding the plastid specific form of phosphoglucomutase, $P g m-p$, exhibited linkage with $S, K$, and $W b$ loci mapping near the middle of chromosome 2 . The relative recombination frequencies in the possible three-point tests suggested that $P g m-P$ was located on the opposite side of $K$ from $W b$. Linkage was not observed between $P g m-p$ and $S k d h$ in family A or between $P g m-p$ and $A r$ in family $\mathrm{G}$.

\section{Chromosome 3}

Leucine aminopeptidase: Both isozymes of LAP are specified by genes located on chromosome 3. The tight linkage between Lap-I and $B$ was demonstrated previously ${ }^{2}$. Our data confirm this observation and indicate that $L a p-l$ is on the centromere side of $B$. The gene coding the LAP-2 isozyme, designated Lap-2, appears to be located near $M$ at the opposite end of the chromosome from $B$. Relatively close linkage ( 9 percent recombination) was observed between $M$ and Lap-2 in family G, and significant deviations from random assortment also were seen between Lap-2 and $s t$ (families $\mathrm{E}$ and $\mathrm{G}$ ) and $A c p-3$ (family $\mathrm{G}$ ). The

Table III. Joint segregation data for pairs of loci exhibiting significant deviations from random assortment

\begin{tabular}{|c|c|c|c|c|c|c|c|c|c|c|c|c|c|}
\hline \multirow[b]{2}{*}{ Loci } & \multirow[b]{2}{*}{ Family } & \multicolumn{9}{|c|}{ No. observed with designated phenotypes* } & \multicolumn{3}{|c|}{ Recomb. } \\
\hline & & $\mathbf{N}$ & $-1-$ & $-/ \mathrm{H}$ & $-1+$ & $\mathrm{H} /-$ & $\mathrm{H} / \mathrm{H}$ & $\mathrm{H} /+$ & $+1-$ & $\overline{+/ \mathrm{H}}$ & $+/+$ & fract. & SE \\
\hline \multicolumn{14}{|l|}{ Chromosome 1} \\
\hline Pur:Idh & A & 63 & 15 & 2 & 0 & & & & 1 & 32 & 13 & 5 & \\
\hline$D: I d h$ & G & 40 & 9 & 1 & 0 & & & & 1 & 19 & 10 & 5 & \\
\hline \multicolumn{14}{|l|}{ Chromosome 2} \\
\hline Oh:Aat-m & $\mathbf{A}$ & 84 & 15 & 6 & 2 & & & & 13 & 33 & 15 & 27 & \\
\hline$O h: S k d h$ & A & 83 & 23 & 0 & 0 & & & & 0 & 41 & 19 & $\leq 1$ & \\
\hline$S k d h: A a t-m$ & A & 84 & 15 & 9 & 4 & 6 & 29 & 4 & 2 & 4 & 11 & 24 & \\
\hline Aat-m:Aldo-p & A & 76 & 18 & 5 & 1 & 10 & 24 & 3 & 0 & 4 & 11 & 17 & \\
\hline Skdh:Aldo-p & A & 75 & 7 & 9 & 3 & 15 & 20 & 3 & 6 & 4 & 8 & 42 & \\
\hline$A r: A a t-m$ & G & 34 & 1 & 3 & 4 & & & & 6 & 16 & 4 & 30 & \\
\hline$A r: S k d h$ & $G$ & 34 & 5 & 3 & 0 & & & & 3 & 13 & 10 & 19 & \\
\hline$S k d h: A a t-m$ & $G$ & 40 & 0 & 2 & 6 & 3 & 15 & 2 & 6 & 6 & 0 & 18 & 8 \\
\hline$S: P g m-p$ & A & 83 & 13 & 2 & 0 & & & & 10 & 43 & 15 & 16 & \\
\hline$W b: P g m-p$ & A & 84 & 17 & 5 & 0 & & & & 6 & 40 & 16 & 13 & 9 \\
\hline$K: P g m-p$ & $\mathbf{A}$ & 84 & 22 & 4 & 0 & & & & 0 & 42 & 16 & 4 & \\
\hline$S: W b$ & A & 84 & 15 & & 0 & & & & 7 & & 62 & 7 & \\
\hline$S: K$ & $\mathbf{A}$ & 84 & 14 & & 1 & & & & 11 & & 58 & 14 & \\
\hline$W b: K$ & $\mathbf{A}$ & 84 & 18 & & 4 & & & & 7 & & 55 & 13 & \\
\hline$W b: P g m-p$ & $\mathrm{E}$ & 109 & 16 & 13 & 1 & & & & 9 & 39 & 31 & 23 & .5 \\
\hline$K: P g m-p$ & E & 108 & 22 & 9 & 0 & & & & 3 & 42 & 32 & 11 & 2 \\
\hline$W b: K$ & E & 115 & 23 & & 7 & & & & 10 & & 75 & 15 & \\
\hline
\end{tabular}

Chromosome 3

\begin{tabular}{|c|c|c|c|c|c|c|c|c|c|c|c|c|c|}
\hline St:Acp-3 & A & 66 & 12 & 5 & 1 & & & & 5 & 25 & 18 & 19 & 5.3 \\
\hline St:B & A & 83 & 13 & & 9 & & & & 7 & & 54 & 21 & 5.1 \\
\hline St:Lap-I & A & 83 & 2 & 5 & 15 & & & & 23 & 31 & 7 & 20 & 4.8 \\
\hline$A c p-3: L a p-1$ & A & 41 & 1 & 4 & 6 & 6 & 7 & 2 & 5 & 8 & 2 & 36 & 7.0 \\
\hline$B: A c p-3$ & A & 67 & 11 & 4 & 1 & & & & 6 & 27 & 18 & 19 & 5.2 \\
\hline B:Lap-I & $\mathbf{A}$ & 57 & 0 & 0 & 12 & & & & 19 & 25 & 1 & 2 & 1.5 \\
\hline St:Lap-2 & $\mathbf{E}$ & 86 & 6 & 12 & 9 & & & & 20 & 26 & 13 & 43 & 6.5 \\
\hline$S t: B$ & $E$ & 116 & 20 & & 16 & & & & 11 & & 69 & 25 & 4.8 \\
\hline M:Lap-2 & G & 37 & 0 & 0 & 6 & & & & 8 & 20 & 3 & 9 & 4.9 \\
\hline$M: A c p-3$ & G & 34 & $\mathbf{0}$ & 2 & 4 & & & & 6 & 18 & 4 & 20 & 7.6 \\
\hline Lap-2:Acp-3 & G & 34 & 2 & 5 & 1 & 3 & 11 & 4 & 1 & 3 & 4 & 35 & 7.6 \\
\hline St:Lap-2 & G & 39 & 3 & 6 & 1 & & & & 5 & 15 & 9 & 37 & 9.2 \\
\hline St:Acp-3 & $G$ & 36 & 7 & 3 & 0 & & & & 0 & 17 & 9 & 9 & 5.0 \\
\hline St:Lap-I & G & 40 & $\mathbf{I}$ & 3 & 8 & & & & 8 & 15 & 5 & 24 & 7.6 \\
\hline St:B & G & 39 & 7 & & 4 & & & & 6 & & 22 & 27 & 8.6 \\
\hline$A c p-3: L a p-1$ & G & 36 & 1 & 2 & 4 & 3 & 11 & 6 & 5 & 3 & 1 & 30 & 6.8 \\
\hline$B: A c p-3$ & G & 35 & 3 & 6 & 1 & & & & 3 & 14 & 8 & 34 & 9.4 \\
\hline B:Lap-1 & G & 39 & 0 & 1 & 12 & & & & 9 & 17 & 0 & 2 & 1.8 \\
\hline
\end{tabular}

- Designations: recessive phenotype or homozygous slow $=-$; heterozygous $=\mathrm{H}$; dominant phenotype or homozygous fast $=+$ 


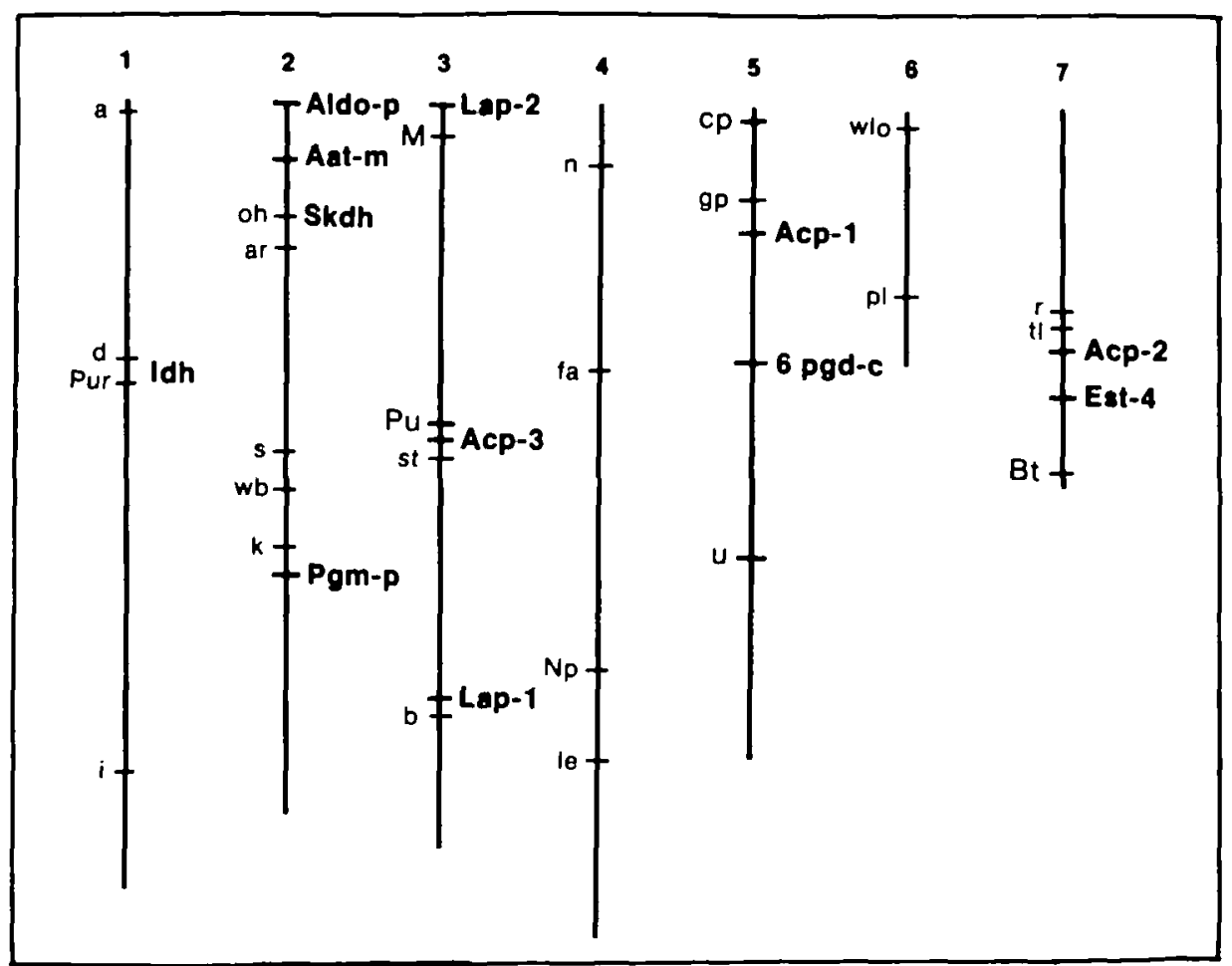

FIGURE 4 Relative map positions on the seven chromosomes of Pisum sativum of the marker loci and the 12 isoxyme loci examined in this study. Marker loci are shown to the left of the chromosome while the iroxyme loci are labeled in bold print to the right of the chromosome.

fewer number of recombinants observed between $M$ and $A C P-3$ than between Lap-2 and $A c p-3$ (family G, Table III) suggests that Lap-2 is situated distal to $M$ on this arm of chromosome 3.
Acid phosphatase-3: This isozyme also is coded by a locus on chromosome 3 . The locus is close to $S t$, near the middle of the known linkage map for this chromosome. The data place $A c p-3$ on the $M$ side of $S t$. Both $A c p-3$

Table III. Continued

\begin{tabular}{|c|c|c|c|c|c|c|c|c|c|c|c|c|c|}
\hline \multirow[b]{2}{*}{ Loci } & \multirow[b]{2}{*}{ Family } & \multicolumn{9}{|c|}{ No. observed with designated phenotypes* } & \multicolumn{3}{|c|}{ Recomb. } \\
\hline & & $\mathbf{N}$ & $-1-$ & $-/ \mathrm{H}$ & $-1+$ & $\mathrm{H} /-$ & $\mathrm{H} / \mathrm{H}$ & $\mathrm{H} /+$ & $+1-$ & $+/ \mathrm{H}$ & $+1+$ & fract. & SE \\
\hline \multicolumn{14}{|l|}{ Chromosome 5} \\
\hline$G_{p: A c p-1}$ & A & 83 & 16 & 4 & 0 & & & & 4 & 33 & 26 & 10 & 3.4 \\
\hline Gp:бpgd-c & A & 84 & 12 & 8 & 0 & & & & 10 & 31 & 23 & 23 & 5.1 \\
\hline $6 p g d-c: A c p-1$ & A & 84 & 17 & 5 & 1 & 4 & 31 & 4 & 0 & 1 & 21 & 10 & 2.4 \\
\hline$G p: A c p-I$ & $\mathrm{E}$ & 115 & 21 & 9 & 2 & & & & 6 & 48 & 29 & 17 & 3.8 \\
\hline$G p: 6 p g d-c$ & E & 116 & 17 & 13 & 2 & & & & 12 & 43 & 29 & 26 & 4.6 \\
\hline $6 p g d-c: A c p-1$ & E & 116 & 21 & 8 & 0 & 6 & 41 & 9 & 0 & 9 & 22 & 15 & 2.6 \\
\hline $6 p g d-c: A c p-1$ & G & 40 & 7 & 4 & 0 & 1 & 19 & 2 & 0 & 2 & 5 & 12 & 3.9 \\
\hline \multicolumn{14}{|l|}{ Chromosome 7} \\
\hline$R: T I$ & A & 83 & 19 & 0 & 0 & & & 0 & 3 & 37 & 24 & 4 & 2.2 \\
\hline$R: A c p-2$ & A & 73 & 0 & 4 & 13 & & & & 21 & 29 & 6 & 15 & 4.5 \\
\hline$T l: A c p-2$ & A & 75 & 0 & 5 & 17 & 9 & 23 & 1 & 12 & 5 & 3 & 19 & 3.6 \\
\hline$R: T l$ & $\mathbf{E}$ & 110 & 26 & 0 & 0 & & & & 2 & 52 & 30 & 2 & 1.1 \\
\hline$R: A c p-2$ & E & 105 & 1 & 6 & 19 & & & & 28 & 41 & 10 & 18 & 4.2 \\
\hline$R: E s t-4$ & E & 101 & 16 & 9 & 0 & & & & 12 & 38 & 26 & 22 & 4.6 \\
\hline$T l: A c p-2$ & E & 107 & 2 & 7 & 19 & 3 & 39 & 8 & 24 & 3 & 2 & 15 & 2.7 \\
\hline$T l: E s t-4$ & E & 108 & 17 & 10 & 0 & 8 & 32 & 10 & 3 & 10 & 18 & 23 & 3.4 \\
\hline$B t: A c p-2$ & E & 105 & 5 & 16 & 12 & & & & 24 & 31 & 17 & 40 & 5.8 \\
\hline$B t: E s t-4$ & $E$ & 105 & 16 & 14 & 4 & & & & 12 & 36 & 23 & 32 & 5.3 \\
\hline Acp2:Est-4 & G & 27 & 0 & 0 & 7 & 0 & 9 & 1 & 5 & 3 & 2 & 16 & 5.5 \\
\hline
\end{tabular}

\section{Chromosome 5}

Acid phosphatase-1: The gene coding the most anodal of the acid phosphatases exhibited nonrandom assortment with $G p$ on chromosome 5 in both populations $A$ and $E$. The recombination frequencies observed between these two loci in both crosses were very similar (Table III).

6-Phosphogluconate dehydrogenase: The subunits of the cytosolic isozyme of 6PGD-2 also are specified by a gene linked with $G p$. Both three-point crosses involving the loci $G p$, $A c p-1$, and $6 p g d-c$ indicate a gene order of $G p-A c p-1-6 p g d-c$. Again, recombination frequencies between 6pgd-c and the other two loci were quite repeatable. An absence of linkage between $6 p g d-c$ and $C p$ in population E suggests that both $6 p g d-c$ and $A c p-1$ lie on the opposite side of $G p$ from $C p$. Preliminary data indicating linkage between $6 p g d-c$ and $F s^{32}$ are consistent with such a position for 6pgd-c.

\section{Chromosome 7}

Acid phosphatase-2: In leaf tissue this locus produces an isozyme of relatively weak activity. The pattern of segregation at $A c p-2$ closely followed segregation seen at the $T l$ and $R$ loci on chromosome 7 .

Esterase-4: This locus also exhibited linkage with $T I$ and $R$. In a four-point cross with $T I$, $R$, and $A c p-2$, the Est-4 locus appeared to be farther from the $T I-R$ region than $A c p-2$ (recombination frequencies: $E s t-4, T I=23$ percent; $A c p-2, T I=15$ percent). The sequence of $T l$ and $R$ relative to $A c p-2$ could not be reliably determined from the data due to the tight linkage between the former two loci; however, Est-4 also showed linkage with $B t$ located near the lower end of the chromosomal linkage map, indicating that $A c p-2$ and Est-4 are located between $T I$ and $B t$ (Figure 4).

\section{Discussion}

Our results provide approximate map locations of 11 previously unmapped isozyme loci in Pisum and confirm the map position of $L a p-1$, the only previously mapped isozyme locus. The consistency of the results in three independent crosses indicated that the deviations from random assortment were not caused by irregularities at meiosis or pseudolinkage due to heterogeneity of chromosome structure between parental lines. Similar to results in 
maize 9 , the isozyme loci in the pea do not appear to cluster within the genome. Not only are the 12 loci distributed over five chromosomes but the map distances between loci on the same choromosome appear to be relatively large. The most closely linked enzyme loci, $A c p-I$ and $6 p g d-c$, showed a recombination frequency of 10 percent while the three loci on chromosome 3 (Lap-1, Acp-3, and Lap-2) span nearly the entire linkage group. In contrast, Tanksley and Rick reported that nearly 30 percent of the isozyme loci in the tomato were associated into tight clusters ${ }^{25}$.

The arrangement of the isozyme loci on chromosomes 2 and 3 are especially fortunate, for nearly every gene on these chromosomes will exhibit linkage with an isozyme marker. This distribution should prove extremely useful for mapping and for marking characters that are difficult or inconvenient to score directly.

Although the four loci coding mitochondrial or plastic specific enzymes or isozymes are all located on chromosome 2 , the possibility that such a grouping reflects a complex of such loci is incompatible with other results. The significant distances between the loci precludes the possibility that these genes may be under the control of a single cis-acting regulator. In addition, two other loci coding plastid specific proteins, $A a t-p$ and $6 p g d-p$, do not exhibit linkage with markers on chromosome 2 or with each other (unpub. data). Loci coding mitochondrial specific enzymes in maize also appear not to be linked ${ }^{15}$.

The designations used for loci specifying mitochondrial and plastid specific isozymes represents a deviation from the terminology used in maize and the tomato 9.18 . Such a change was initiated because recent studies have demonstrated that the subcellular compartmentation of isozymes in many systems (e.g., phosphoglucomutase, aspartate aminotransferase, malate dehydrogenase, and glucose phosphate isomerase) is an important and predictable characteristic of these systems (see reviews by Gottlieb ${ }^{12}$, Newton ${ }^{15}$, and Weeden ${ }^{29}$ ). Since comparison of linkage groups can only be accomplished when homologous loci are being considered it is imperative to determine the subcellular local- ization of isozymes, especially those known to have organelle specificities, and to conveniently mark their localization. The designation of loci coding plastid specific isozymes with a suffix "p", cytosolic with a "c" and mitochondrial with an " $\mathrm{m}$ " would serve this purpose. Standard numbering protocol could be used to distinguish multiple isozymes in the same subcellular compartment.

\section{References}

1. AlLARD, R. W. Formulas and tables to facilitate the calculation of recombination values in heredity. Hilgardia 2:235-279. 1956.

2. ALMGARD, G. and K. OHLUND. Inheritance and location of a biochemical character in Pisum. Pisum Newsl. 2:9. 1970.

3. BASSIRI, A. and M. W. ADAMS. An electrophoretic survey of seedling isozymes in several Phaseolus species. Euphytica 27:447-459. 1978.

4. Bender, K., M. Nagel, and E. Gunther. Est-6, a further polymorphic esterase in the rat. Blochem. Genet. 20:221-229. 1982

5. BLIXT, S. The pea. In Handbook of Genetics, vol. 2. R. C. King Ed. Plenum Press, New York. p. 181-221. 1974

6. Brown, A. H. D. Barley. In Isozymes in Plant Genetics and Breoding, part B. S. D. Tanksley and T. J. Orton, Eds. Elsevier, Amsterdam. p. 57-77. 1983.

7. Cardy, B. J., C. W. Stuber, and M. M. GoOdman. Techniques for starch gel electrophoresis of enzymes from maize (Zea mays L.). Dept. of Statistics Mimeo Series No. 1317, North Carolina State Univ., Raleigh. 1980.

8. Clayton, J. W. and D. N. Tretiak. Aminecitrate buffers for $\mathrm{pH}$ control in starch gel electrophoresis. J. Fish. Res. Bd. Can. 29:1169-1172. 1972.

9. GOOdMAN, M. M., C. W. STUber, and K. J. NEWTON. Isozyme loci in maize. In Maize for Biological Research. W. F. Sheridan, Ed. Plant Molecular Biology Association, Charlottesville, Virginia. p. 53-60. 1982.

10. Gorman, M. B. and Y. T. KIANG. Variety-specific electrophoretic variants of four soybean enzymes. Crop Sci. 17:963-965. 1977.

11. of genetic variation within the named soybean germplasm. Genetics 100:s27.1982.

12. GoTTLIEB, L. D. Conservation and duplication of isozymes in plants. Science 216:373-380. 1982.

13. HART, G. E. Hexaploid wheat. In Isozymes in Plant Genetics and Breeding, part B. S. D. Tanksley and T. J. Orton, Eds. Elsevier, Amsterdam. p. 35-56. 1983.

14. - and N. A. TULEeN. Chromosomal locations of eleven Elytrigia elongata (- Agropyron elongatum) isozyme structural genes. Genet.Res. 41: 181-202. 1983.
15. Newton, K. J. Genetics of mitochondrial isozymes. In Isozymes in Plant Genetics and Breeding, part A. S. D. Tanksley and T. J. Orton, Eds. Elsevier, Amsterdam. p. 157-174. 1983.

16. Przybylska, J., H. PARzySz, and S. Blixt. Electrophoretic study of several enzyme systems of genus Pisum with reference to its taxonomy. Pisum Newsl. 13:42-43. 1981.

17. QUIROS, C. F. Alfalfa, Luzern. In Isozymes in Plant Genetics and Breeding, part B. S. D. Tankstey and T. J. Orton, Eds. Eksevier, Amsterdam. p. 253-294. 1983.

18. Rick, C. M. Tomato. In Isozymes in Plant Genetics and Breeding, part B. S. D. Tanksley and T. J. Orton, Eds. Elsevier, Amsterdam. p. 147-155. 1983.

19. Scandalios, J. G. Isozymes: genetic and biochemical regulation of alcohol dehydrogenase. In Regulation of Enzyme Synthesis and Activity in Higher Plants. H. Smith, Ed. Academic Press, NY. p. 129-153. 1977.

20. - and L. G. ESPIRITU. Mutant aminopeptidases in Pisum satioum I. Developmental genetics and chemical characteristics. Mol. Gen. Genet. 105:101-112. 1969.

21. SElandeR, R. K., M. H. SMIth, S. Y. YANG, W. E. JOHNSON, and J. B. GENTRY. Biachemical polymorphism and systematics in the genus Peromyscus. I. Variation in the old-field mouse (Peromyscus polionotus). Unio. Texas Publ. 7103:49-90. 1971.

22. Shaw, C. R. and R. PRASAD. Starch gel electrophoresis - a compilation of recipes. Biochem. Genet. 4:297-320. 1970.

23. Simcox, P. D. and D. T. Dennis. 6-Phosphogluconate dehydrogenase isozymes from the developing endosperm of Rlcinus communis L. Plant Physiol. 62:287-290. 1978.

24. TANKSLEY, S. D. and T. J. ORTON. Isozymes in Plant Genetics and Breeding. Elsevier, Amsterdam. 1983.

25. - and C. M. RICK. Isozymic linkage map of the tomato: applications in genetics and breeding. TAG 57:161-170. 1980.

26. WALL, J. R. and S. W. WALL. Isozyme polymorphisms in the study of evolution in the Phaseolus oulgaris-P. coccineus complex of Mexico. In Isozymes, vol. 4. C. L. Markert, Ed. Academic Press, NY. p. 287-305. 1975.

27. WEEDEN, N. F. lsozyme variation at selection loci in Pisum. Pisum Newsl. 15:58-59. 1983.

28. - Pea 6-phosphogluconate dehydrogenase isozymes. Pisum Newsl. 15:56-58. 1983.

29. —_. Plastid isozymes. In Isozymes in Plant Genetics and Breeding, part A. S. D. Tanksley and T. J. Orton, Eds. Elsevier, Amsterdam. p. 139-156. 1983.

30. - Distinguishing among white seeded bean cultivars by means of allozyme genotypes. Euphytica 33:199-208. 1984.

31. _ and L. D. GotTLIEB. The genetics of chloroplast enzymes. J. Hered. 71:392-396. 1980.

32. and G. A. MARX. Linkage determinations for several isozymic loci in Pisum. Pisum Newsl. 15:54-55. 1983. 